\title{
ON SPECIAL CLASSES OF ENTIRE FUNCTIONS WHOSE ZEROS AND GROWTH ARE RESTRICTED
}

\author{
BY
}

CARL L. PRATHER

\begin{abstract}
The present paper is an investigation of the uniform limits on bounded sets of entire functions of genus $<2 p$ whose zeros are real, or lie in an even number of sectors (or correspondingly on rays) of a certain size, both the number and size depending on $p$. A characterization of the uniform limits of entire functions of genus $<2 p$ whose zeros lie in these sectors is given.
\end{abstract}

Introduction. A real entire function is one which is real on the real axis. We will consider extensions of the classical problem of approximating functions $f \neq 0$ uniformly on discs $|z| \leqslant R$ by a sequence of real polynomials whose roots are real.

One such extension was made by Hellerstein and Korevaar [1] who investigated the class of holomorphic functions $f \neq 0$ which are uniform limits on $|z| \leqslant R$ of a sequence of entire functions of genus $\leqslant 2 p$, for any integer $p>0$, which are (constant multiples of a) real function with real zeros or more generally having their zeros in $S_{\alpha}$, a double sector (adjoining sectors) which forms an angular neighborhood of the real axis, defined by

$$
S_{\alpha}=[z:|\arg z| \leqslant \alpha,|\arg z-\pi| \leqslant \alpha] .
$$

When the functions are constant multiples of real entire functions with real zeros (when $\alpha \in(0, \pi /(4 p+4)))$, they show that this class of functions consists precisely of (the restrictions of) real entire functions of the form

$$
f(z)=\exp \left(-a z^{2 p+2}\right) F(z),
$$

where $a>0$ and $F(z)$ is (a constant multiple of) a real entire function of genus $<(2 p+1)$ whose zeros are real (respectively, consists of restrictions of functions of the form (I), for which $|\arg a| \leqslant(2 p+1) \alpha$ and $F(z)$ is an entire function of genus $<(2 p+1)$ whose zeros lie in $\left.S_{\alpha}\right)$.

For the case of real entire functions with only real zeros, use the notation of Hellerstein and Williamson [2] and denote the class by $V_{2 p}$. The class $V_{0}$ is the Laguerre-Pólya class, where Laguerre [9] and Pólya [11] characterized these as being the functions $\neq 0$ which are uniformly approximable on compact sets in the plane by real polynomials whose zeros are real. The latter result is a generalization of the classical results of G. Pólya and E. Lindwart [12] that deal with uniform limits of polynomials whose zeros lie in sectors.

Received by the editors December 2, 1977 and, in revised form, March 5, 1979.

AMS (MOS) subject classifications (1970). Primary 30A82, 30A64; Secondary 30A66.

Key words and phrases. Real entire functions, finite genus, uniform approximation on bounded sets (discs). 
In $\S 1$, we determine the class of uniform limits on bounded sets of entire functions of genus $\leqslant 2 p$ whose zeros lie in a number of multiple double sectors, each sector having the same size. The number of sectors and the size of each sector depends on $p$. We characterize such a class and show that the sizes of the sectors are critical in that the functions in the class change as soon as the sectors assume a critical size. This is a generalization of the result of Hellerstein and Korevaar stated above where the zeros lie in one double sector.

This paper contains results which are part of a dissertation written at Northwestern University under the direction of Professor R. P. Boas, Jr. The author expresses his thanks to the editor for the suggestions on revising.

0. Preliminary results. We now present a well known lemma of Korevaar [8, Lemma 3.3, p. 69], or [1, Lemma 3, p. 223] which on several occasions we shall utilize.

Lemma 0.1. Let $k$ and $m$ be positive integers and let $C=|C| \exp (-\operatorname{im} \beta)$, for $\beta$ real, be an arbitrary complex number. Then uniformly on every bounded set

$$
\begin{aligned}
\left\{( 1 - z / k e ^ { i \beta } ) \operatorname { e x p } \left(\left(z / k e^{i \beta}\right)+\cdots\right.\right. & \left.\left.+z^{m-1} /(m-1)\left(k e^{i \beta}\right)^{m-1}\right)\right\}^{\left[m k^{m}|C|\right]} \\
& \rightarrow \exp \left(-C z^{m}\right) \text { as } k \rightarrow \infty
\end{aligned}
$$

Here $[x]$ denotes the greatest integer $\leqslant x$.

Throughout, we will denote the phrase "uniformly on bounded sets" by U.B.S. It is also standard to denote the expression

$$
(1-z / \beta) \exp \left(z / \beta+(1 / 2)(z / \beta)^{2}+\cdots+(1 / m)(z / \beta)^{m}\right)
$$

by $E(z / \beta, m)$ and we will comply with this throughout. Also, unless otherwise stated, we will be concerned with limit functions $f \neq 0$.

1. Approximation by entire functions whose zeros lie in multiple sectors. The problem of approximation by polynomials whose zeros lie in a number of sectors is an old one, first attacked by E. Lindwart and G. Pólya [12]. Extensive generalizations have been given, principally by $J$. Korevaar and his students [6]. In particular, Korevaar and J. Lange [8] consider the case of approximation by polynomials whose zeros lie in radial sets or more generally by entire functions whose genus is restricted by the location of the zeros.

Here we will consider approximation by entire functions whose genus is not restricted by the location of the zeros (which Korevaar and Lange do) but instead the location of the zeros will be restricted by the given genus of the approximating functions. Specifically, we consider approximation by entire functions of genus $<2 p$ whose zeros lie in $2 r$ sectors of a certain size about the origin where $r$ divides $p$. Our Theorem 1.1 is a generalization of the following theorem of Hellerstein and Korevaar [1, p. 222]:

Let $\beta<\pi /(4 p+4)$ and denote by $S_{\beta}$ the closed double sector

$$
S_{\beta}=\{z:|\arg z| \leqslant \beta,|\arg z-\pi|<\beta\} .
$$


Consider the class of holomorphic functions $f \neq 0$ which are U.B.S. limits of entire functions of genus $\leqslant 2 p$ whose zeros lie in $S_{\beta}$. Then this class consists of the restrictions of entire functions of the form

$$
f(z)=\left[\exp \left(-a z^{2 p+2}\right)\right] F(z),
$$

with $|\arg a|<(2 p+1) \beta$ and $F$ is an entire function of genus $\leqslant(2 p+1)$ whose zeros lie in $S_{\beta}$.

Generalizing the above theorem, we prove the following theorem:

THEOREM 1.1. Let $\left\{f_{n}(z)\right\}_{n=1}^{\infty}$ be a sequence of entire functions of genus $<2 p$; for each $n$, let $f_{n}(z)$ have zeros $\left\{\alpha_{n k}\right\}_{k=1}^{\infty}$ lying in $r$ closed double sectors equally distributed about the origin, each sector having angle size less than $\pi /((2 r)(p+1))$ where $r$ divides $p$. Then a necessary and sufficient condition that $\left\{f_{n}(z)\right\}_{n=1}^{\infty}$ be convergent U.B.S. to an entire function $f(z) \neq 0$ is that $f$ have the form $f(z)=$ $\exp \left(-a z^{2 p+2 r}\right) \cdot F(z)$, where $F(z)$ is an entire function of genus $<2 p+2 r-1$ having zeros $\left\{\alpha_{k}\right\}_{k=1}^{\infty}$ lying in the sectors stated and where the constant a satisfies $|\arg a|<$ $2(p+1) \alpha<\pi / 2 r, \quad\left(\alpha<\pi /(4 r(p+1))\right.$. Precisely, the zeros $\left\{\alpha_{n k}\right\}_{k=1}^{\infty}, n=$ $1,2, \ldots$, lie in the following $r$ closed double sectors $\cup_{t=0}^{r-1} S_{t}$, where

$$
\begin{aligned}
S_{t}=\{z:|\arg z-t \pi / r| \leqslant \alpha<\pi /(4 r(p+1)), \\
|\arg z-((t \pi / r)+\pi)| \leqslant \alpha<\pi /(4 r(p+1))\} .
\end{aligned}
$$

Proof. Necessity. Let $\left\{f_{n}(z)\right\}_{n=1}^{\infty}$ be given with zeros $\left\{\alpha_{n k}\right\}_{k=1}^{\infty}, n=1,2, \ldots$, stated as above. Since $r$ divides $p, p=r k$, so that $2 p=2 r k$, for some integer $k$. We also write

$$
f_{n}(z)=\exp \left(Q_{n}(z)\right) \prod_{k=1}^{\infty} E\left(z / \alpha_{n k}, 2 p\right)
$$

where $Q_{n}(z)=a_{n, 2 p} z^{2 p}+a_{n, 2 p-1} z^{2 p-1}+\cdots+a_{n, 1} z$ and for each $n$, $\sum_{k=1}^{\infty} 1 /\left(\alpha_{n k}\right)^{2 p+1}<\infty$.

Let $1, \omega, \ldots, \omega^{r-1}$ be the $r$ th roots of unity. Then consider $g_{n}(z)=f_{n}(z) \cdot f_{n}(\omega z)$ $\cdots \cdot f_{n}\left(\omega^{r-1} z\right)$. It is a standard result that $g_{n}$ becomes

$$
g_{n}(z)=\exp \left(r\left(a_{n, r} z^{r}+a_{n, 2 r} z^{2 r}+\cdots+a_{n, 2 p} z^{2 p}\right)\right) \prod_{l=1}^{\infty} E\left(\left(z / \alpha_{n l}\right)^{r}, 2 k\right) .
$$

We now replace $z^{r}$ by $w$ and consider the functions

$$
h_{n}(w)=\exp \left[r\left(a_{n, r} w+\cdots+a_{n, 2 p} w^{2 k}\right)\right] \prod_{l=1}^{\infty} E\left(w /\left(\alpha_{n l}\right)^{r}, 2 k\right)
$$

so that $h_{n}\left(w^{r}\right)=g_{n}(w)$. Now the sequence $\left[h_{n}(w)\right]_{n=1}^{\infty}$ has genus $<2 k$, for all $n$ and each $h_{n}(w)$ has zeros $\left\{\left(\alpha_{n l}\right)^{r}\right\}_{n=1}^{\infty}$ which lie in the closed double sector

$$
S_{\alpha}=\{z:|\arg w| \leqslant r \alpha<\pi / 4(p+1),|\arg w-\pi|<r \alpha\} .
$$

Thus the theorem of Hellerstein and Korevaar applies and $\left\{h_{n}(w)\right\}_{n=1}^{\infty}$ converges U.B.S. to an entire function of the form $h(w)=\exp \left(-b w^{2 k+2}\right) \cdot H(w)$, where $H(w)$ is an entire function of genus $\leqslant 2 k+1$ with zeros lying in $S_{\alpha}$. By Hurwitz's theorem we know that the zeros of $h(w)$ are points of accumulation of the zeros 
$\left\{\left(\alpha_{n l}\right)^{r}\right\}_{l=1}^{\infty}$ of $h_{n}(w)$. Hence some subsequence $\alpha_{n, l}$ satisfies $\left(\alpha_{n, l}\right)^{r} \rightarrow\left(\alpha_{l}\right)^{r}$ as $j \rightarrow \infty$ as well as $\alpha_{n, l} \rightarrow \alpha_{l}$ for a suitable choice of $\alpha_{l}$.

$$
\begin{aligned}
h(w)= & \exp \left(-b w^{2 k+2}+b_{2 k+1} w^{2 k+1}+\cdots+b_{1} w\right) \\
& \cdot \prod_{k=1}^{\infty} E\left(w /\left(\alpha_{k}\right)^{r}, 2 k+1\right) .
\end{aligned}
$$

Moreover, the theorem of Hellerstein and Korevaar give us that the zeros $\left(\alpha_{k}\right)^{r}$ of $h(w)$ satisfy

$$
\sum_{k=1}^{\infty} 1 /\left|\left(\alpha_{k}\right)^{r}\right|^{2 k+2}=\sum_{k=1}^{\infty} 1 /\left|\left(\alpha_{k}\right)\right|^{2 k r+2 r}=\sum_{k=1}^{\infty} 1 /\left|\alpha_{k}\right|^{2 p+2 r}<\infty
$$

and the coefficient $b$ of $w^{2 k+2}$ in the polynomial of the exponent in (*) satisfies

$$
|\arg b|<2(p+1) \beta<2(p+1) \pi / 4(p+1)=\pi / 2
$$

(since $\beta=r \alpha<\pi / 4(p+1)$ ). So we have that $f(z) f(\omega z) \cdots \cdot f\left(\omega^{r-1} z\right)=$ $\lim g_{n}(z)=\lim h_{n}\left(z^{r}\right)=h\left(z^{r}\right)$ as $n \rightarrow \infty$.

We want to know the form of $f(z)$. We know that by applying Hurwitz's theorem to $f_{n}(z)$ and $f(z), \lim \alpha_{n, l}=\alpha_{l}$ as $j \rightarrow \infty$. It is easy to see that

$$
\sum_{l=1}^{\infty} 1 /\left|\alpha_{n, l}\right|^{2 p+2 r}<M
$$

for some constant $M$ and all $n_{j}$.

By the theorem of Lindwart and Pólya [12], [1, Lemma 2, p. 222] the subsequence $\left\{f_{n}(z)\right\}_{j=1}$ and hence the convergent sequence $\left\{f_{n}(z)\right\}_{n=1}^{\infty}$ converges U.B.S. to an entire function of genus $\leqslant 2 p+2 r=r(2 k+2)$.

Now, from the fact that

$$
\begin{aligned}
f(z) f(\omega z) \cdots \cdot f\left(\omega^{r-1} z\right)= & \exp \left(-b z^{r(2 k+2)}+b_{r(2 k+1)} z^{r(2 k+1)}+\cdots+b_{r} z^{r}\right) \\
& \cdot \prod_{k=1}^{\infty} E\left(z /\left(\alpha_{k}\right)^{r}, 2 p+r\right)
\end{aligned}
$$

(here $2 p+r=r(2 k+1)$ ), we can express $f(z)$ in the form

$$
\begin{aligned}
f(z)= & \exp \left(-d z^{r(2 k+2)}+d_{r(2 k+2)-1} z^{r(2 k+2)-1}+\cdots+d_{1} z\right) \\
& \cdot \prod_{k=1}^{\infty} E\left(z / \alpha_{k}, r(2 k+2)-1\right) \\
= & \exp \left(-d z^{2 p+2 r}+d_{2 p+2 r-1} z^{2 p+2 r-1}+\cdots+d_{1} z\right) \\
& \cdot \prod_{k=1}^{\infty} E\left(z / \alpha_{k}, 2 p+2 r-1\right)
\end{aligned}
$$

and the zeros $\left\{\alpha_{k}\right\}_{k=1}^{\infty}$ lie in the $r$ closed double sectors given in the statement of the theorem and satisfy $\sum_{k=1}^{\infty} 1 /\left(\alpha_{k}\right)^{2 p+2 r}<\infty$. Also the coefficient $d$ of $z^{2 p+2 r}$ in the polynomial of the exponent satisfies $|\arg d|<2(p+1) \alpha<\pi / 2 r$.

Sufficiency. For notation, denote by $V_{2 p+2 r}(\alpha)$ for $\alpha \in(0, \pi / 4 r(p+1))$, the class of entire functions of the form $\exp \left(-a z^{2 p+2 r}\right) h(z)$, where $h$ is entire of genus $<2 p+2 r-1$ with zeros in the sectors $\cup_{t=0}^{r-1} S_{t}$, where $S_{t}$ is as in the statement of the theorem. Now let $f$ belong to $V_{2 p+2 r}(\alpha)$ with the form $f(z)=\exp \left(-a z^{2 p+2 r}\right) F(z)$ 
where the constant $a$ satisfies $|\arg (+a)|<2(p+1) \alpha<\pi / 2 r$. We show that $f$ can be approximated U.B.S. by a sequence of entire functions of genus $<2 p$ whose zeros lie in the indicated sectors.

Using Korevaar's Lemma 0.1 , first let $m=2 p+1$. Every complex number $-b$ can be written in the form $-b=C_{1}+C_{2}+\cdots+C_{2 r}$, where $C_{j}=\left|C_{j}\right| e^{-i \varphi_{j}}$ and $\left|\varphi_{j}\right|<2(p+1) \alpha$ or $\left|\varphi_{j}-\pi\right| \leqslant 2(p+1) \alpha(\bmod 2 \pi)$ for $\alpha>0$. Since products of functions in the class $V_{2 p+2 r}(\alpha)$ also belong to $V_{2 p+2 r}(\alpha)$, we get that $\exp \left(b z^{2 p+1}\right) \in$ $V_{2 p+2 r}(\alpha)$ for every complex number $b$. Next, let $m=(2 p+2)$ in the lemma. All functions of the form $\exp \left(b_{1} z+\cdots+b_{2 p+1} z^{2 p+1}\right) \in V_{2 p+2 r}(\alpha)$ and U.B.S. limits of functions in $V_{2 p+2 r}(\alpha)$ are in $V_{2 p+2 r}(\alpha)$, we get that $\exp \left(b_{2 p+2} z^{2 p+2}\right) \in$ $V_{2 p+2 r}(\alpha)$. Continue in this fashion, letting $m=k$, for $k=(2 p+3), \ldots,(2 p+$ $2 r-1)$ and get $\exp \left(b_{k} z^{k}\right) \in V_{2 p+2 r}(\alpha)$. Finally, let $m=2 p+2 r$. Since functions of the form $\exp \left(b_{1} z+\cdots+b_{2 p+2 r-1} z^{2 p+2 r-1}\right) \in V_{2 p+2 r}(\alpha)$ and likewise for U.B.S. limits of functions $V_{2 p+2 r}(\alpha)$, we get $\exp \left(-a z^{2 p+2 r}\right) \in V_{2 p+2 r}(\alpha)$ where $|\arg (+a)|$ $<2(p+1) \alpha<\pi / 2 r$.

Combining these results, we get that

$$
\begin{aligned}
f_{M}(z)= & A z^{m} \exp \left(-a z^{2 p+2 r}\right) \exp \left(b_{1} z+\cdots+b_{2 p+2 r-1} z^{2 p+2 r-1}\right) \\
& \cdot \prod_{n<M}\left(1-z / z_{n}\right) \exp \left(\left(z / z_{n}\right)+\cdots+(1 /(2 p+2 r-1))\left(z / z_{n}\right)^{2 p+2 r-1}\right)
\end{aligned}
$$

belongs to $V_{2 p+2 r}(\alpha)$. If $f$ has a finite number of zeros, then the proof is complete. If $f$ has infinitely many zeros, then $f$ is the U.B.S. limit of the $f_{M}$ as $M \rightarrow \infty$.

COROLlARY 1.2. Let $\left\{f_{n}(z)\right\}_{n=1}^{\infty}$ be a sequence of entire functions of genus $<2 p$; for each $n$, let $f_{n}(z)$ have zeros $\left\{\alpha_{n k}\right\}_{k=1}^{\infty}$ which lie on the rays which pass through the $2 r$ th roots of unity, expressible as $\cup_{m=0}^{r-1} R_{m}$, where $R_{m}=\{z: \arg z=m \pi / r$ or $\arg z=m \pi / r+\pi\}$, where $r$ divides $p$. Then a necessary and sufficient condition that $\left\{f_{n}(z)\right\}_{n=1}^{\infty}$ be convergent U.B.S. to an entire function $f(z)$ is that $f(z)$ be an entire function of the form

$$
f(z)=\exp \left(-a z^{2 p+2 r}\right) F(z)
$$

where $F(z)$ is entire of genus $\leqslant(2 p+2 r-1)$ and where $\arg a=0$ i.e. $a>0$ and the zeros $\left\{\alpha_{k}\right\}_{k=1}^{\infty}$ of $F(z)$ lie on the given $2 r$ rays and $\sum_{k=1}^{\infty} 1 /\left|\alpha_{k}\right|^{2 p+2 r}<\infty$.

This is the special case $\alpha=0$ of the theorem just proved.

COROllary 1.3 OF THEOREM 1.1. The theorem remains true if we allow the sequence $\left\{f_{n}(z)\right\}_{n=1}^{\infty}$ of entire functions to have the property that, for all $n$, at most $T$ (independent of $n$ ) zeros of $f_{n}(z)$ do not lie in the r-closed double sectors $\cup_{m=0}^{r-1} S_{m}$ as in the theorem. The limit function $f(z)$ will be a polynomial of degree $<T$ multiplied by the function obtained in the statement of the theorem.

The estimate on the angle size $\alpha=\pi / 4 r(p+1)$ is critical in Theorem 1.1 in a sense analogous to $\alpha=\pi / 4(p+1)$ being the critical angle in the theorem of Hellerstein and Korevaar. The following result is analogous to one of Hellerstein and Korevaar [1, Theorem 3, p. 222]. 
THEOREM 1.4. Let $r$ divide $p$ and suppose that $\alpha>\pi / 4 r(p+1)$. Then the class of holomorphic functions $f \neq 0$ which are limits on $|z|<R$ of entire functions of genus $<2 p$ whose zeros lie in $\cup_{m=0}^{r-1} S_{m}$ consists of all holomorphic functions $\neq 0$ in $|z|<R$ whose zeros lie in $\cup_{m=0}^{r-1} S_{m}$.

Here $S_{m}=\{z:|\arg (z)-m \pi / r| \leqslant \alpha,|\arg z-((m+r) \pi / r)| \leqslant \alpha\}$.

We now take up the question of what happens when the sectors assume a certain critical size, considering first $\alpha=\pi / 4(p+1)$.

It is remarked in [1] that one could formulate a theorem in the critical case of a single double sector. Since it is not done explicitly there, we include it here for completeness and follow it with a theorem for the critical case in our more general setting.

Proposition 1.5. Let $\left\{f_{n}(z)\right\}_{n=1}^{\infty}$ be a sequence of entire functions of genus $<2 p$, each function having zeros which lie in the double sector $\{z:|\arg z|<\pi / 4(p+1)$, $|\arg z-\pi|<\pi / 4(p+1)\}=S$ and suppose that $\left\{f_{n}(z)\right\}_{n=1}^{\infty}$ converges U.B.S. to a limit function $f \neq 0$. Then $f(z)$ is entire of the form

$$
\begin{array}{r}
f(z)=B z^{m} \exp \left(a_{1} z+\cdots+a_{p+1} z^{p+1}+\cdots+a_{2 p+2} z^{2 p+2}+\cdots\right. \\
\left.+a_{3 p+3} z^{3 p+3}+\cdots+a_{4 p+4} z^{4 p+4}\right) \cdot F(z)
\end{array}
$$

where $F(z)=\prod_{k=1}^{\infty} E\left(z / \alpha_{k}, 4 p+3\right)$, and $m$ is an integer $>0$, the zeros $\alpha_{k}$ lie in the aforesaid double sector, $\sum_{k=1}^{\infty} 1 /\left|\alpha_{k}\right|^{4 p+4}<\infty$ and $\sum_{k=1}^{\infty} \operatorname{Re}\left(\left(1 / \alpha_{k}\right)^{2 p+2}\right)$ converges to a sum $\leqslant-(2 p+2) \operatorname{Re}\left(a_{2 p+2}\right), a_{4 p+4}$ is real and $\geqslant 0$.

Before proving this, we go back to the case when the approximation is being done by polynomials. In his thesis, Korevaar proves the following theorem ([4, Theorem 9.1]. See also [5, p. 590, 3rd paragraph]):

Denote by $U$ the double sector $\{z:|\arg z| \leqslant \pi / 4,|\arg z-\pi| \leqslant \pi / 4\}$. Suppose that a sequence of polynomials $\left\{p_{n}(z)\right\}_{n=1}^{\infty}$ converges U.B.S. to a limit function $f \neq 0$ and the zeros of each $p_{n}(z)$ belong to $U$. The limit function $f$ can be expressed in the form

$$
z^{m} \exp \left(a_{0}+a_{1} z+\cdots+a_{4} z^{4}\right) \cdot \prod_{n=1}^{\infty} E\left(z / z_{n}, 3\right),
$$

where $m \geqslant 0, m$ an integer, $z_{n} \in U, \sum_{n=1}^{\infty} 1 /\left|z_{n}\right|^{4}<\infty, \Sigma_{n} \operatorname{Re}\left(\left(1 / z_{n}\right)^{2}\right)$ converges to a sum $<-2 \operatorname{Re}\left(a_{2}\right)$ and $a_{4} \geqslant 0$.

We give now the proof of Proposition 1.5. Say

$$
F_{n}(z)=\exp \left(a_{n 2 p} z^{2 p}+\cdots+a_{n 1} z\right) \prod_{k=1}^{\infty} E\left(z / \alpha_{n k}, 2 p\right),
$$

where $\alpha_{n k} \in S$.

Let $1, \lambda, \ldots, \lambda^{p}$ the $(p+1)$ st roots of unity. Then, when computed,

$$
F_{n}\left(z^{p+1}\right)=f_{n}(z) f_{n}(\lambda z) \cdots \cdot f_{n}\left(\lambda^{p} z\right)
$$

becomes $\prod_{k=1}^{\infty} E\left(z^{p+1} /\left(\alpha_{n k}\right)^{p+1}, 1\right)$. Let $w=z^{p+1}$. Then $F_{n}(w)=$ $\prod_{k=1}^{\infty} E\left(w /\left(\alpha_{n k}\right)^{p+1}, 1\right)$ which now have their zeros in $U$. Moreover, $F_{n}(w)$ has genus 1 and by hypothesis is U.B.S. convergent. By Korevaar's theorem for 
polynomials, each $F_{n}(w)$ is a limit U.B.S. of polynomials $\left\{p_{n j}(z)\right\}_{j=1}^{\infty}$ whose zeros lie in $U$. Thus, the sequence $\left\{p_{n j}(z)\right\}_{j=1}^{\infty}, n=1,2, \ldots$ is U.B.S. convergent and by Korevaar's theorem again, $\lim _{n j} p_{n j}(w)=\lim _{n} F_{n}(w)=F(w)$ and $F(w)$ is expressible in the form

$$
F(w)=A z^{m} \exp \left(a_{1} z+\cdots+a_{4} z^{4}\right) \prod_{n=1}^{\infty} E\left(z / z_{n}, 3\right)
$$

where $m>0, m$ an integer, $z_{n} \in U, \sum_{n=1}^{\infty} 1 /\left|z_{n}\right|^{4}<\infty$, and $\sum_{n=1}^{\infty} \operatorname{Re}\left(\left(1 / z_{n}\right)^{2}\right)$ converges to a sum $<-2 \operatorname{Re}\left(a_{2}\right)$, and $a_{4}>0$.

Therefore,

$$
\begin{aligned}
F\left(z^{p+1}\right) & =f(z) f(\lambda z) \cdots \cdot f\left(\lambda^{p} z\right) \\
= & A z^{m} \exp \left(a_{1} z^{p+1}+a_{4} z^{2 p+2}+a_{3} z^{3 p+3}+a_{4} z^{4 p+4}\right) \prod_{j=1}^{\infty} E\left(z^{p+1} /\left(\alpha_{j}\right)^{p+1}, 3\right)
\end{aligned}
$$

and so for some $i=0, \ldots, p, f\left(\lambda^{i} z\right)$ can be expressed in the form

$$
B z^{m} \exp \left(b_{0} z+\cdots+b_{p+1} z^{p+1}+\cdots+b_{4 p+4} z^{4 p+4}\right) \prod_{j=1}^{\infty} E\left(z / \alpha_{j}, 4 p+3\right)
$$

where now the $\alpha_{j}$ lie in $S$ and where $b_{p+1}=a_{1} /(p+1), b_{2 p+2}=a_{2} /(p+1)$, $b_{3 p+3}=a_{3} /(p+1)$, and $b_{4 p+4}=a_{4} /(p+1)$. Furthermore, $\sum_{j=1}^{\infty} \operatorname{Re}\left(\left(1 / \alpha_{j}\right)^{2 p+2}\right)$ converges to a sum $<-2 \operatorname{Re}\left((p+1)\left(b_{2 p+2}\right)\right)=-(2 p+2) \operatorname{Re}\left(b_{2 p+2}\right), a_{4 p+4}>0$ and $\sum_{j=1}^{\infty} 1 /\left|\alpha_{j}\right|^{4 p+4}<\infty$. This proves 1.5 .

We also state the critical case when the zeros of the approximating functions lie in the multiple sectors of a critical size. This time $\alpha=\pi / 4 r(p+1)$ when the zeros lie in $\cup_{m=0}^{r-1} T_{m}$, where

$$
T_{m}=\{z:|\arg z-m \pi / r|<\pi / 4 r(p+1),|\arg z-((m \pi / r)+\pi)|<\pi / 4 r(p+1)\} .
$$

Proposition 1.6. Let $\left\{f_{n}(z)\right\}_{n=1}^{\infty}$ be a sequence of entire functions of genus $<2 p$, each function having zeros which lie in $\cup_{m=0}^{r-1} T_{m}$ as above, where $r$ divides $p$. Suppose that $\left\{f_{n}(z)\right\}_{n=1}^{\infty}$ converges U.B.S. to a limit function $f \neq 0$. Then $f(z)$ is entire of the form

$$
f(z)=A z^{m} \exp (Q(z)) \prod_{k=1}^{\infty} E\left(z / \alpha_{k}, 4 p+4 r-1\right),
$$

where $Q(z)=b_{1} z+\cdots+b_{4 p+4 r} z^{4 p+4 r}$, and the zeros $\alpha_{k}$ lie in $\cup_{m=0}^{r-1} T_{m}$, satisfying $\sum_{k=1}^{\infty} 1 /\left|\alpha_{k}\right|^{4 p+4 r}<\infty$. The coefficient $b_{2 p+2 r}$ satisfies $\sum_{k=1}^{\infty} \operatorname{Re}\left(\left(1 / \alpha_{k}\right)^{2 p+2 r}\right)$ converges to a sum which is $<-(2 p+2) \operatorname{Re}\left(b_{2 p+2 r}\right)$ and $b_{4 p+4 r}>0$.

Proof. Since $r$ divides $p, p=r l$ for some integer $l$. We let

$$
f_{n}(z)=\exp \left(a_{n 1} z+\cdots+a_{n 2 p} z^{2 p}\right) \prod_{k=1}^{\infty} E\left(z / \alpha_{n k}, 2 p\right) .
$$

Let $1, \omega, \ldots, \omega^{r-1}$ be the $r$ th roots of unity. Consider $g_{n}(z)=f_{n}(z)(\omega z)$ $\cdots \cdot f_{n}\left(\omega^{r-1} z\right)$. The usual computation shows that this is equal to

$$
\exp \left(r\left(a_{n, r} z^{r}+a_{n, 2 r} z^{2 r}+\cdots+a_{n, r(2 l)}\left(z^{r}\right)^{2 l}\right)\right) \prod_{k=1}^{\infty} E\left(z^{r} /\left(\alpha_{k}\right)^{r}, 2 l\right) \text {. }
$$


Letting $w=z^{r}$, we consider

$$
h_{n}(w)=\exp \left(r\left(a_{n, r} w+a_{n, 2 r} w^{2}+\cdots+a_{n, 2 p} w^{2 l}\right)\right) \prod_{k=1}^{\infty} E\left(w /\left(\alpha_{k}\right)^{r}, 2 l\right) .
$$

Now $\left[h_{n}(w)\right\}_{n=1}^{\infty}$ is a sequence of entire functions of genus $2 l$ whose zeros lie in the two critical double sectors

$$
S=\{z:|\arg z|<\pi / 4(p+1),|\arg z-\pi|<\pi / 4(p+1)\} .
$$

Also $\left\{h_{n}(w)\right\}_{n=1}^{\infty}$ converges U.B.S. since $\left\{f_{n}(z)\right\}_{n=1}^{\infty}$ does.

By the last proposition, we have that $h_{n}(w)$ has as limit an entire function $h(w)$ which is of the form

$$
h(w)=A w^{s} \exp \left(a_{1} w+a_{2} w^{2}+\cdots+a_{4 l+4} w^{4 l+4}\right) \prod_{k=1}^{\infty} E\left(w /\left(\alpha_{k}\right)^{r}, 4 l+3\right),
$$

where the constant $a_{2 l+2}$ satisfies the condition that

$$
\sum_{k=1}^{\infty} \operatorname{Re}\left(\left(1 / \alpha_{k}\right)^{2 p+2 r}\right) \equiv \sum_{k=1}^{\infty} \operatorname{Re}\left(\left(1 / \alpha_{k}\right)^{r(2 l+2)}\right)
$$

converges to a sum $<-(2 p+2) \operatorname{Re}\left(a_{2 l+2}\right)$.

Now

$$
\begin{aligned}
h\left(z^{r}\right) & =f(z) f(\omega z) \cdots \cdot f\left(\omega^{r-1} z\right) \\
& =A z^{r s} \exp \left(a_{1} z^{r}+a_{2} z^{2 r}+\cdots+a_{4 l+4} z^{4 p+4 r}\right) \prod_{k=1}^{\infty} E\left(z^{r} /\left(\alpha_{k}\right)^{r}, 4 l+3\right)
\end{aligned}
$$

(because $4 p+4 r=4 l r+4 r$ ). Note that the canonical product $\Pi_{k=1}^{\infty} E\left(z^{r} /\left(\alpha_{k}\right)^{r}, 4 l+3\right)$ can also be expressed as

$$
\begin{gathered}
\prod_{k=1}^{\infty}\left(1-\left(z / \alpha_{k}\right)^{r}\right) \exp \left(\left(z / \alpha_{k}\right)^{r}+\cdots+1 /(4 l+3)\left(z / \alpha_{k}\right)^{4 p+3 r}\right) \\
=\prod_{k=1}^{\infty} E\left(\left(z / \alpha_{k}\right)^{r}, 4 p+3 r\right) \quad(\text { since } 4 l r+3 r=4 p+3 r)
\end{gathered}
$$

Therefore, for some $i=0, \ldots, r-1, f\left(\omega^{i} z\right)$ can be expressed in the form

$$
A z^{m} \exp \left(b_{1} z+\cdots+b_{4 p+4 r} z^{4 p+4 r}\right) \prod_{k=1}^{\infty} E\left(z / \alpha_{k}, 4 p+4 r-1\right)
$$

where the zeros $\alpha_{k}$ satisfy $\sum_{k=1}^{\infty} 1 /\left|\alpha_{k}\right|^{4 p+4 r}<\infty$. Furthermore the coefficient $b_{2 p+2 r}$ satisfies $\sum_{k} \operatorname{Re}\left(1 / \alpha_{k}\right)^{2 p+2 r}$ converges to a sum which is $<-(2 p+$ 2) $\operatorname{Re}\left(b_{2 p+2 r}\right)$ and $b_{4 p+4 r}>0$.

In recent work [3, Theorems 1 and 2], Hellerstein and Shea have given results showing that entire functions of finite genus whose zeros are real or lie along rays emanating from the origin have positive Nevalinna deficiencies at the origin and moreover the deficiency tends to infinity as the genus tends to infinity. Their results are applicable to the functions given in Corollary 1.2. 


\section{BIBLIOGRAPHY}

1. S. Hellerstein and J. Korevaar, Limits of entire functions whose zeros and growth are restricted, Duke Math. J. 30 (1963), 221-227.

2. S. Hellerstein and J. Williamson, Derivatives of entire functions and a question of Pólya, Trans. Amer. Math. Soc. 227 (1977), 227-249.

3. S. Hellerstein and D. Shea, Minimal deficiencies for entire functions with radially distributed zeros, Proc. London Math. Soc. 37 (1978), 35-55.

4. J. Korevaar, The zeros of polynomials approximating to an entire function and its canonical representation, thesis, University of Leiden, 1949.

5. The zeros of approximating polynomials and the canonical representation of an entire function, Duke Math. J. 18 (1951), 573-592.

6. L_ Limits of polynomials whase zeros lie in a given set, Entire Functions and Related Parts of Analysis, Proc. Sympos. Pure Math., Amer. Math. Soc., Providence, R. I., 1968, pp. 261-272.

7. J. Korevaar and C. Loewner, Approximation on an arc by polynomials with restricted zeros, Nederl. Akad. Wetensch. Proc. Ser. A 67 (1964), 121-128.

8. J. Korevaar and J. E. Lange, Limits of polynomials whose zeros lie in a radial set, Trans. Amer. Math. Soc. 114 (1965), 65-79.

9. E. Laguerre, Sur les fonctions d genre zero et du genre un, Oeuvres 1 (1898), 174-177.

10. B. Ja. Levin, Distribution of the zeros of entire functions, Trans. Math. Monographs, Vol. 5, Amer. Math. Soc., Providence, R. I., 1964.

11. G. Pólya, Über Annäherung durch Polynome mit Lauter Reelen Wurzeln, Rend. Circ. Mat. Palermo 36 (1913), pp. 279-295 = Collected Works, Location of zeros, Vol. 2, M.I.T. Press, Cambridge, Mass., 1974, pp. 54-70.

12. G. Pólya and E. Lindwart, Über einen Zusammenhang zwischen der Konvergenz von Polynomen folgen und der Verteilung ihrer Wurzeln, Rend. Circ. Mat. Palermo 37 (1914), pp. 297-304 = Collected Works, Location of zeros, Vol. 2, M.I.T. Press, Cambridge, Mass. 1974, pp. 54-70.

Department of Mathematics, Virginia Polytechnic Institute and State Universtty, BlacksBURG, VIRGINIA 24061 